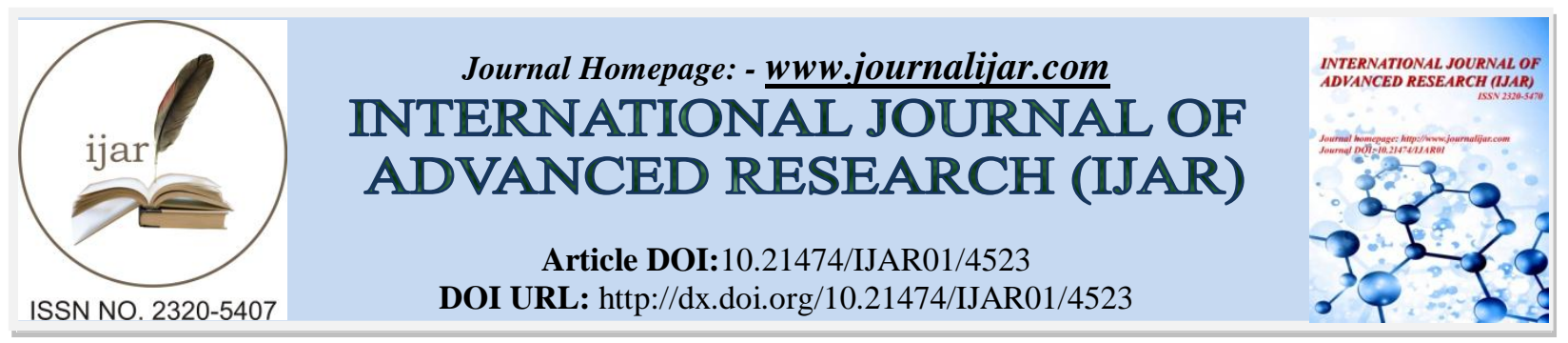

RESEARCH ARTICLE

\title{
PRODUCTION AND MARKETING OF RIDGE GOURD IN MEERUT DISTRICT OF WESTERN UTTAR
} PRADESH.

\author{
Krishna Kant ${ }^{1}$ and Subhash Chandra ${ }^{2}$. \\ 1. Research Scholar, Department of Agril. Economics, N.D. University of Agriculture and Tech., Faizabad, U.P., \\ India \\ 2. Research Investigator, A.E.R. Centre, University of Delhi, India
}

\section{Manuscript Info}

Manuscript History

Received: 18 April 2017

Final Accepted: 20 May 2017

Published: June 2017

Key words:-

Costs and returns, resource use efficiency, price spread and marketing efficiency

\section{Abstract}

Uttar Pradesh occupies second place in area about 12.1 per cent of total vegetables production during 2012-13. Meerut district of western Uttar Pradesh was selected purposively in order to avoid operational in convenience. On an average gross income and net incomes of ridged gourd were worked out as Rs. 200559 and 82689. Input-output ratio was worked out as $1: 1.70$. The producer's share in consumer's rupee was worked out to be $97.45,65.66$ and 63.72 per cent marketing cost accounted i.e. Rs.65, 445 and 459 per qt., in Channel - I, II and III. However, middlemen's margin was Rs. 00.00, 877and 947 per quintal in Channel-II, and III. In respect of marketing efficiency Channel I (39.33 per cent) was found most efficient over other channels because none of middleman was engaged in this channel. Suggestive policy implications to overcome the production and marketing constraints of vegetables are that farmers be trained through designated extension agency by participation directly and be updated about latest farming technology and management of vegetables crop. Input supply should be linked with co-operative marketing system to help the producers $\&$ traders.

Copy Right, IJAR, 2017,. All rights reserved.

\section{Introduction:-}

Ridge gourd (Luffa acutangula) is one of the important cucurbitaceous vegetables grown commercially throughout India .It grows very well in a warm hot climate, the optimum temperature being250C-300C. India is the second largest producer of the vegetables (162.19 Million tonnes) in the world, contributing 14.0 per cent of the total world production of vegetables (FAO, 2014). Across different states of the country, Uttar Pradesh occupies second place in area with 326.2 million hectares of land under cultivation and production (5176.1 Million tonnes) with about 12.1 per cent of total vegetables production during 2012-13, (Indian, Horticulture Database, 2013). Uttar Pradesh has been bestowed with wide range of climatic and physio-graphical conditions, surplus family labour, and marginal land holdings. The cultivation of vegetables provides higher return per unit area in less time than foodgrains besides providing nutritive food. Vegetable farming being labour intensive also offers more employment opportunities. Vegetables are an important cash crop in the Western Uttar Pradesh. Therefore to explore the possibilities of raising farm production and farm income in this region, there is a need to understand the economics of vegetables cultivation. In Western Uttar Pradesh, Meerut district occupies an important place in terms of area and production of 
vegetables cultivation. Vegetables were grown on an area of 15621 hectare, with its production of 306.95 thousand tonnes and productivity 19.65 quintal/ha (DHO, Uttar Pradesh, 2015-16). Vegetable is an intensive input utilization crop and input requirement varies from region to region and farmer to farmer. Marketing facilities and vegetables prices has improved during the last decade thereby attracting the more cultivators to grow vegetable but they are facing continuous increasing input price such as labour charges, diesel prices, fertilizer prices, availability of inputs at proper time.

Hence there is need to change the farming system favouring adoption of vegetable farming which can help in generating additional income and employment for the farmers. At the same time the marketing operations due to seasonality of produce have crucial role in deciding the profit of the farmers on the one hand and the level of availability to consumers on other hand (Singh et.al. 2015). Keeping in view the importance of the crop, the study entitled "Production and marketing of ridge gourd of Meerut district of western Uttar Pradesh" is proposed with the following specific objectives:

1. To workout costs and returns and examine the resource use efficiency of ridge gourd on sample farms.

2. To identify marketing channels and to assess marketing costs, marketing margins, producer's share in the consumer's rupees and marketing efficiency on sample farms.

3. To examine constraints in production and marketing of ridge gourd.

\section{Research Methodology:-}

The district Meerut was selected purposively to avoid the operational inconvenience of the investigator. A multistage stratified random cum purposive sampling technique was used for the present study. Out of twelve blocks in Meerut district, one block namely Kharkoda having highest area under vegetables crop was selected purposively. A list of all the villages falling under the selected block was prepared and arranged in ascending order according to the area covered by vegetables crop therefore; five villages were selected randomly from the list. A list of vegetables growers of the five selected villages was prepared along with their size of holdings. Then from this list the farm holdings were categorized into three size groups i.e. marginal (below 1 ha), small (1 to 2 ha.) and medium ( 2 to 4 ha.) and a sample of hundred respondents were selected using proportionate random sampling technique. The secondary data were collected from published/ unpublished record of district and block headquarters. The primary data were collected through well structured pre - tested schedule of enquiry by interview method during the agricultural year 2015-16.

\section{Selection of Market:-}

Meerut mandi where most of the vegetables of study area are being disposed off as such leading market was selected for the study of marketing aspects. A sizeable number of intermediaries were interviewed for assessing the imperfections of vegetables marketing system.

\section{Analytical Tools:-}

Suitable statistical tools were used for analysis of data. Tabular analysis was used to compare the different parameters among marginal, small and medium size groups of the farmers. To examine the profitability, the cost of cultivation and returns were worked out on per ha basis. In this computation weighted average were used. Cost of cultivation and returns were estimated using standardised CACP cost concept.

\section{Regression Analysis:-}

To study the effect of various independent variables on the dependent variables, various forms of production function were explored. However, Cobb-Douglas production function, elasticity of production and return to scale, was found to be best fit for the analysis of data. The mathematical form of Cobb-Douglas function is as follows: $\mathrm{Y}=\mathrm{ax}_{1}{ }^{\mathrm{b}_{1}} \cdot \mathrm{x}_{2} \mathrm{~b}_{2} \ldots \ldots \ldots \ldots \ldots \ldots \mathrm{Xn}^{\mathrm{bn}}$

Where,

$\mathrm{Y}=$ Dependent variable (output value in rupees/ha)

$\mathrm{X}_{1}=\mathrm{i}^{\text {th }}$ independent variable (input value rupees/ha)

$\mathrm{a}=$ Constant

$\mathrm{b}_{1}=$ Production elasticity with respect to $\mathrm{X}_{\mathrm{i}}$ 's

The value of the constant (a) and coefficient (bi) in respect of independent variable in the function have been estimated by using the method of least square. The Cobb-Douglas production function in log form is as follows: 
$\log Y=\log a+b_{1} \log X_{1}+b_{2} \log X_{2}+b_{3} \log X_{3}+b_{4} \log X_{4}+U$

Where,

$\begin{array}{lll}\mathrm{Y} & = & \text { Value of gross returns of crops (Rs./ha) } \\ \mathrm{X}_{1} & = & \text { Expenditure on human labour (Rs./ha) } \\ \mathrm{X}_{2} & = & \text { Expenditure on seed (Rs./ha) } \\ \mathrm{X}_{3} & = & \text { Expenditure on manures and fertilizers (Rs./ha) } \\ \mathrm{X}_{4} & = & \text { Expenditure on plant protection (Rs./ha) } \\ \mathrm{a} & = & \text { Intercept } \\ \mathrm{U} & =\text { Error term }\end{array}$

\section{Marginal Value Product (MVP):-}

The marginal values of product inputs were estimated by the following formula:

Where,

$$
\text { (MVP) } x_{j}=b_{j} \frac{\bar{Y}}{\bar{X}_{j}}
$$

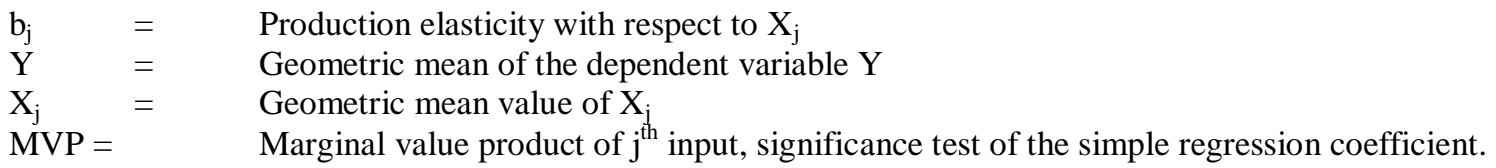

\section{Price Spread:-}

The difference between the price paid by the consumer and the net price received by producer was taken as the concept of price spread. This included not only the actual prices at various stages of marketing channels, but also the costs incurred in the process of the movement of the produce from the farm to the consumer and the margin of the various intermediaries. The model prices at different levels were obtained to work out the gross margins of various agencies. The deduction of the costs incurred by the concerned agencies from the gross margins gave rise to net margins.

\section{Marketing Efficiency:-}

Marketing efficiency was analysed by using the following Shepherd's formula. Higher the ratio, higher the efficiency and vice-versa.

$$
\text { Marketing efficiency }(\mathrm{ME})=\frac{\mathrm{V}}{\mathrm{I}}-1
$$

Where,

$\mathrm{V}=$ Value of goods sold (Consumer's price)

$\mathrm{I}=$ Total marketing costs $(\mathrm{MC})$

\section{Result Discussion:-}

The results to costs and returns, resource use efficiency, price spread and marketing efficiency of ridge gourd vegetable cultivation has been worked out and are discussed as under.

\section{Cost of cultivation and returns from Ridged Gourd:-}

Per hectare costs on various input factors in ridged gourd production were worked out. The details of input costs are shown in Table 1. On an average, the cost of cultivation of ridged gourd per hectare came to Rs. 117872 . The cost of cultivation was maximum on medium farms followed by marginal and small farms. Per hectare cost of cultivation was highest on medium farms, mainly due to maximum investment on working capital compared to the marginal and small farms. On an average the study further reveals that major components on which maximum cost was incurred were human labour, plant protection, manures and fertilizer, irrigation, machinery charges and bullock labour with $22.95,13.18,8.10,7.84,3.84,3.30$ and 0.24 per cent, respectively. A similar trend indicated on all categories of sample farms. The costs incurred on interest on working capital, rental value of land, interest on fixed capital and 13\% managerial cost of sub-total were calculated as 1.49, 20.87, 6.69 and 11.52 per cent of total costs, respectively. The maximum share among these costs was rental value of owned land being 20.87 per cent of total cost per hectare. 
Table 1:-Input used on different size of sample farms of Ridged gourd

(Rs./ha)

\begin{tabular}{|c|c|c|c|c|c|}
\hline \multirow[t]{2}{*}{ S. No. } & \multirow[t]{2}{*}{ Components of investment } & \multicolumn{4}{|c|}{ Size of farms } \\
\hline & & Marginal & Small & Medium & Overall \\
\hline 1. & Human labour & $\begin{array}{c}27168 \\
(23.14)\end{array}$ & $\begin{array}{l}26183 \\
(22.67)\end{array}$ & $\begin{array}{c}27801 \\
(23.04)\end{array}$ & $\begin{array}{c}27050 \\
(22.95)\end{array}$ \\
\hline a. & Family labour & $\begin{array}{c}24812 \\
(21.14)\end{array}$ & $\begin{array}{c}16288 \\
(14.10)\end{array}$ & $\begin{array}{c}6214 \\
(5.15) \\
\end{array}$ & $\begin{array}{c}15771 \\
(13.38)\end{array}$ \\
\hline b. & Hired labour & $\begin{array}{l}2356 \\
(2.01) \\
\end{array}$ & $\begin{array}{c}9895 \\
(8.57) \\
\end{array}$ & $\begin{array}{c}21587 \\
(17.89) \\
\end{array}$ & $\begin{array}{l}11279 \\
(9.57) \\
\end{array}$ \\
\hline 2. & Bullock labour & $\begin{array}{c}428 \\
(0.36)\end{array}$ & $\begin{array}{c}298 \\
(0.26) \\
\end{array}$ & $\begin{array}{c}121 \\
(0.10)\end{array}$ & $\begin{array}{c}282 \\
(0.24)\end{array}$ \\
\hline 3. & Machinery charges & $\begin{array}{c}3650 \\
(3.11)\end{array}$ & $\begin{array}{c}3905 \\
(3.38) \\
\end{array}$ & $\begin{array}{c}4097 \\
(3.40)\end{array}$ & $\begin{array}{c}3884 \\
(3.30)\end{array}$ \\
\hline 4. & Seed & $\begin{array}{c}8264 \\
(7.04)\end{array}$ & $\begin{array}{r}9452 \\
(8.18) \\
\end{array}$ & $\begin{array}{l}10884 \\
(9.02)\end{array}$ & $\begin{array}{c}9553 \\
(8.10)\end{array}$ \\
\hline 5. & Manure \& fertilizers & $\begin{array}{c}8648 \\
(7.37) \\
\end{array}$ & $\begin{array}{c}9255 \\
(8.01) \\
\end{array}$ & $\begin{array}{c}9826 \\
(8.14) \\
\end{array}$ & $\begin{array}{c}9243 \\
(7.84) \\
\end{array}$ \\
\hline 6. & Irrigation charges & $\begin{array}{l}4740 \\
(4.03) \\
\end{array}$ & $\begin{array}{c}4798 \\
(4.15) \\
\end{array}$ & $\begin{array}{c}4051 \\
(3.36) \\
\end{array}$ & $\begin{array}{l}4529 \\
(3.84) \\
\end{array}$ \\
\hline 7. & Plant protection & $\begin{array}{c}14849 \\
(12.65) \\
\end{array}$ & $\begin{array}{c}15527 \\
(13.44) \\
\end{array}$ & $\begin{array}{c}16248 \\
(13.47) \\
\end{array}$ & $\begin{array}{c}15541 \\
(13.18) \\
\end{array}$ \\
\hline 8. & Total working capital & $\begin{array}{c}67747 \\
(57.71)\end{array}$ & $\begin{array}{c}69418 \\
(60.10)\end{array}$ & $\begin{array}{c}73028 \\
(60.53)\end{array}$ & $\begin{array}{c}70062 \\
(59.44)\end{array}$ \\
\hline 9. & Interest on working capital & $\begin{array}{c}1693 \\
(1.44)\end{array}$ & $\begin{array}{c}1735 \\
(1.50) \\
\end{array}$ & $\begin{array}{c}1826 \\
(1.51) \\
\end{array}$ & $\begin{array}{c}1752 \\
(1.49) \\
\end{array}$ \\
\hline 10. & Rental value of land & $\begin{array}{c}24000 \\
(20.44)\end{array}$ & $\begin{array}{c}24500 \\
(21.21)\end{array}$ & $\begin{array}{c}25300 \\
(20.98)\end{array}$ & $\begin{array}{c}24600 \\
(20.87)\end{array}$ \\
\hline 11. & Interest on fixed capital & $\begin{array}{l}10439 \\
(8.90)\end{array}$ & $\begin{array}{l}6576 \\
(5.70)\end{array}$ & $\begin{array}{c}6613 \\
(5.50) \\
\end{array}$ & $\begin{array}{c}7876 \\
(6.69)\end{array}$ \\
\hline 12. & Sub-total & $\begin{array}{l}103879 \\
(88.49)\end{array}$ & $\begin{array}{l}102229 \\
(88.50)\end{array}$ & $\begin{array}{l}106767 \\
(88.50)\end{array}$ & $\begin{array}{l}104292 \\
(88.48)\end{array}$ \\
\hline 13. & $13 \%$ cost managerial of sub-total & $\begin{array}{c}13504 \\
(11.50) \\
\end{array}$ & $\begin{array}{c}13290 \\
(11.50) \\
\end{array}$ & $\begin{array}{c}13879 \\
(11.50) \\
\end{array}$ & $\begin{array}{c}13580 \\
(11.52) \\
\end{array}$ \\
\hline 14. & Grand total & $\begin{array}{c}117383 \\
(100.00)\end{array}$ & $\begin{array}{c}115519 \\
(100.00)\end{array}$ & $\begin{array}{c}120646 \\
(100.00)\end{array}$ & $\begin{array}{c}117872 \\
(100.00)\end{array}$ \\
\hline
\end{tabular}

Note- Figure in parentheses shows the percent to corresponding total.

\section{Measure of costs and returns of Ridged gourd:-}

Main product of ridged gourd yield was calculated as 74.50, 79.28 and 85.89 quintal and average yield 79.22 quintal per hectare. Gross returns was calculated as maximum in medium farms Rs. 223314 followed by small, marginal farms and observed average gross return Rs. 200559, net income per hectare was found highest in medium farms Rs. 102668 and lowest in marginal farm and small farms, net income over cost $\mathrm{C}_{1}$ medium farm is Rs. 141847, followed by small and marginal farms, respectively and net return over cost $C_{2}$ is highest medium farm Rs.116547 followed by small and marginal farms, respectively. The B: C ratio was found to be the highest in ridged gourd crop medium farms 1: 1.85 followed by small farms $1: 1.71$ and marginal farms $1: 1.53$, respectively.

Per hectare gross income was observed maximum under medium farms i.e. Rs. 223314 followed by small and marginal farms, respectively. Gross income was highest on medium farms due to higher investment on H.Y.V. of seeds resulted higher productivity. On an overall average, gross income came to Rs. 200559 where as net income was Rs. 82689 per hectare. Overall average, family labour income, farm investment income and farm business income were worked out to be Rs. 112040, 128743 and 144516 per hectare, respectively. Cost of production per quintal of ridged gourd was computed to be Rs. 1576, 1457, and 1405 on marginal, small and medium farms, respectively with an average of Rs. 1488. Average input-output ratio on cost $\mathrm{A}_{1}, \mathrm{~B}_{1}, \mathrm{~B}_{2}, \mathrm{C}_{1}, \mathrm{C}_{2}$ and $\mathrm{C}_{3}$ were worked out and came to $1: 3.57,1: 3.13,1: 2.26,1: 2.51,1: 1.92$ and $1: 1.70$, respectively. Input-output ratio related to cost 
$\mathrm{C}_{3}$ was highest on medium farms followed by small farms, and marginal farms. In respect to cost $\mathrm{A}_{1}$, Input-output ratio cost $A_{1}$, was highest on marginal farms (1:4.03) followed by small and medium farms, respectively (Table 2).

Table 2:- Measures of cost and returns of Ridged gourd

\begin{tabular}{|c|c|c|c|c|c|}
\hline \multirow{2}{*}{$\begin{array}{c}\text { S. } \\
\text { No. }\end{array}$} & \multirow{2}{*}{ Particulars } & \multicolumn{4}{|c|}{ Size of farms } \\
\hline & & Marginal & Small & Medium & Overall \\
\hline 1. & Cost $A_{1}$ & 44628.00 & 54865.00 & 68640.00 & 56043.00 \\
\hline 2. & Cost $\mathrm{B}_{1}$ & 55067.00 & 61441.00 & 75253.00 & 63919.00 \\
\hline 3. & Cost $\mathrm{B}_{2}$ & 79067.00 & 85941.00 & 100553.00 & 88519.00 \\
\hline 4. & Cost $C_{1}$ & 79879.00 & 77729.00 & 81467.00 & 79690.00 \\
\hline 5. & Cost $\mathrm{C}_{2}$ & 103879.00 & 102229.00 & 106767.00 & 104290.00 \\
\hline 6. & Cost $C_{3}$ & 117383.00 & 115519.00 & 120646.00 & 117870.00 \\
\hline 7. & Product (qt./ha) & 74.50 & 79.28 & 85.89 & 79.22 \\
\hline 8. & Price of Product (qt./ha) & 2485.00 & 2500.00 & 2600.00 & 2528.00 \\
\hline 9. & Gross Income & 180162.00 & 198200.00 & 223314.00 & 200559.00 \\
\hline 10 & Net return over cost $C_{1}$ & 100283.00 & 120471.00 & 141847.00 & 120869.00 \\
\hline 11. & Net return over cost $C_{2}$ & 76283.00 & 95971.00 & 116547.00 & 96269.00 \\
\hline 12. & Net income & 62779.00 & 82681.00 & 102668.00 & 82689.00 \\
\hline 13. & Family labour income & 101095.00 & 112259.00 & 122761.00 & 112040.00 \\
\hline 14 & Farm investment income & 110722.00 & 127047.00 & 148460.00 & 128743.00 \\
\hline 15. & Farm Business Income & 135534.00 & 143335.00 & 154674.00 & 144516.00 \\
\hline 16 & Cost of production (Rs./q) & 1576.00 & 1457.00 & 1405.00 & 1488.00 \\
\hline 17. & Input-Output Ratio & & & & \\
\hline A & On the basis of cost $A_{1}$ & $1: 4.03$ & $1: 3.61$ & $1: 3.25$ & $1: 3.57$ \\
\hline $\mathrm{B}$ & On the cost ' $\mathrm{B}_{1}$ ' basis & $1: 3.27$ & $1: 3.22$ & $1: 2.96$ & $1: 3.13$ \\
\hline $\mathrm{C}$ & On the cost ' $\mathrm{B}_{2}$ ' basis & $1: 2.27$ & $1: 2.30$ & $1: 2.22$ & $1: 2.26$ \\
\hline $\mathrm{D}$ & On the cost ' $C_{1}$ ' basis & $1: 2.25$ & $1: 2.54$ & $1: 2.74$ & $1: 2.51$ \\
\hline $\mathrm{E}$ & On the cost ' $C_{2}$ ' basis & $1: 1.73$ & $1: 1.93$ & $1: 2.09$ & $1: 1.92$ \\
\hline $\mathrm{F}$ & On the cost ' $\mathrm{C}_{3}$ ' basis & $1: 1.53$ & $1: 1.71$ & $1: 1.85$ & $1: 1.70$ \\
\hline
\end{tabular}

Note- Figure in parentheses shows the percent to corresponding total.

\section{Resource use efficiency of Ridged gourd:-}

The Cobb-Douglas production function was found to be best fit because of high $\mathrm{R}^{2}$ value. The coefficient of multiple determinations $\left(\mathrm{R}^{2}\right)$ on marginal, small and medium size group of farms accounted for $0.84637,0.89981$ and 0.85487 , respectively indicating that all the explanatory variable viz., human labour, seed, manure and fertilizers and plant protection together contributed $84.637,89.981$ and 85.487 per cent of variation of gross income in sample farms, respectively. Significance of factor of production it is observed that on marginal farms, the elasticity of production with respect to human labour seed and manure \& fertilizer were statistically significant at 1 per cent and 5 per cent level of significance that these input factors contributed to the output significantly. In case of small farms, elasticity of production with respect to human labour and seed were found significant at 1 per cent and level of significance, respectively. In case of medium farms, elasticity of production with respect to human labour and seed were found significant at 1 per cent level of significance, respectively. Rest factors of production included in production process were found statistically non-significant. It can be inferred that there was no further scope for application of these input in production of ridged gourd.

Returns to scale on marginal, small and medium farms were analyzed and observed to be $0.81578,0.86141$ and 0.83501 , respectively, which was found to be less than unity. It is therefore, inferred that increasing all factors by one per cent simultaneously results increase of the returns by less than 1 per cent on each farm situation. Sum of elasticises indicates decreasing return to scale.

Marginal value productivities are positive and more than unity in case of human labour, manure \& fertilizer and plant protection on marginal, small and medium farms and in case of seed it was positive on marginal and small farms only and more than unity which indicates scope for increasing the expenditure on this input variable. In case of seed on medium farms it was found less than unity which indicated excess investment on this variable hence, there are need to decrease it, for increasing profitability of farms Table (3). 
Table 3:-Elasticity coefficient of the production function for Ridged gourd.

\begin{tabular}{|c|c|c|c|c|c|c|c|c|c|c|}
\hline \multirow{2}{*}{$\begin{array}{c}\text { Size } \\
\text { group } \\
\text { of } \\
\text { farms }\end{array}$} & \multicolumn{4}{|c|}{ Production elasticities } & \multirow{2}{*}{$\begin{array}{l}\text { Sum of } \\
\text { elastici } \\
\text { tices }\end{array}$} & \multirow[t]{2}{*}{$\mathbf{R}^{2}$} & \multicolumn{4}{|c|}{ Marginal value product } \\
\hline & $\begin{array}{c}\text { Human } \\
\text { Labour } \\
\left(\mathbf{X}_{1}\right)\end{array}$ & $\begin{array}{l}\text { Seed } \\
\left(\mathbf{X}_{2}\right)\end{array}$ & $\begin{array}{c}\text { Manure\& } \\
\text { fertilizers } \\
\left(\mathbf{X}_{3}\right)\end{array}$ & $\begin{array}{c}\text { Plant } \\
\text { Protecti } \\
\text { on } \\
\left(\mathbf{X}_{4}\right)\end{array}$ & & & $\begin{array}{c}\text { Human } \\
\text { Labour } \\
\left(\mathbf{X}_{1}\right)\end{array}$ & $\begin{array}{c}\text { See } \\
\text { d } \\
\left(\mathbf{X}_{2}\right. \\
)\end{array}$ & $\begin{array}{c}\text { Manure \& } \\
\text { fertilizers } \\
\left(\mathbf{X}_{3}\right)\end{array}$ & $\begin{array}{l}\text { Plant } \\
\text { Prote } \\
\text { ction } \\
\left(\mathrm{X}_{4}\right) \\
\end{array}$ \\
\hline $\begin{array}{l}\text { Margin } \\
\text { al }\end{array}$ & $\begin{array}{l}0.1584 * \\
* \\
(0.0429 \\
) \\
\end{array}$ & $\begin{array}{l}0.7492 * \\
* \\
(0.14124 \\
)\end{array}$ & $\begin{array}{l}0.3955^{*} \\
(0.1588)\end{array}$ & $\begin{array}{l}0.02631 \\
0.06813\end{array}$ & $\begin{array}{l}0.8157 \\
8\end{array}$ & $\begin{array}{l}0.84 \\
637\end{array}$ & 1.17 & $\begin{array}{c}3.5 \\
9\end{array}$ & 2.57 & 6.01 \\
\hline Small & $\begin{array}{l}0.2241 * \\
* \\
(0.0579 \\
)\end{array}$ & $\begin{array}{l}0.19128 \\
* * \\
(0.04782 \\
)\end{array}$ & $\begin{array}{l}0.1776 \\
(0.1992)\end{array}$ & $\begin{array}{l}0.0367 \\
0.06372\end{array}$ & $\begin{array}{l}0.8614 \\
1\end{array}$ & $\begin{array}{l}0.89 \\
981\end{array}$ & 1.57 & $\begin{array}{c}4.6 \\
1\end{array}$ & 2.45 & 5.29 \\
\hline $\begin{array}{l}\text { Mediu } \\
\text { m }\end{array}$ & $\begin{array}{l}0.47240 \\
* * * \\
(0.0736 \\
9) \\
\end{array}$ & $\begin{array}{l}0.32141 \\
* * \\
(0.09594 \\
1)\end{array}$ & $\begin{array}{l}0.227477 \\
(0.155431 \\
)\end{array}$ & $\begin{array}{l}0.0811 \\
(0.16458 \\
2)\end{array}$ & $\begin{array}{l}0.8350 \\
1\end{array}$ & $\begin{array}{l}0.85 \\
487\end{array}$ & 3.24 & $\begin{array}{c}0.7 \\
8\end{array}$ & 1.12 & 6.93 \\
\hline
\end{tabular}

(Figures in parentheses show standard error of respective variable)

$* * 1 \%$ level of significance and $* 5 \%$ level of significance.

Price spread, marketing costs, margins and marketing efficiency of ridged gourd:-

Marketing channels are the routes through which the vegetables move from the point of production to ultimate consumer. The length of the channel varies from commodity to commodity. Number of intermediaries was involved in the marketing of ridge gourd were wholesalers, retailers and consumers. Based on the information major channels in marketing of vegetables were identified as follows-

\section{Channel-I (Producer - Consumer):-}

Table 4 indicates that marketing Channel $1^{\text {st }}$ for disposal of ridged gourd was producer - consumer and also indicates that ridged gourd was directly sold to consumer without involvement of any middleman. On an average marketing costs incurred by producer was worked out i.e. Rs. 65.00 per quintal. Producer's share in consumer rupee was 97.45 per cent; it was highest in comparison to other channels. Channel I said to be an efficient channel as share of producer in consumer rupee is highest but the limitation of this channel was that only very small quantity of the total produce was sold through this channel.

Table4:-Price spread for ridged gourd marketing in Channel- I $\quad$ (Rs. /qt.)

\begin{tabular}{|c|c|c|c|c|c|}
\hline \multirow[t]{2}{*}{ S. No. } & \multirow[t]{2}{*}{ Particulars } & \multicolumn{4}{|c|}{ Size of farms } \\
\hline & & Marginal & Small & Medium & Average \\
\hline 1. & Net price received by the producer & $\begin{array}{c}2485.00 \\
(97.56)\end{array}$ & $\begin{array}{c}2500.00 \\
(97.35)\end{array}$ & - & $\begin{array}{l}2492.50 \\
(97.45)\end{array}$ \\
\hline 2. & Cost incurred by the producer & & & & \\
\hline (i) & Grading, filling weighing, stretching etc & $\begin{array}{c}8.00 \\
(0.31)\end{array}$ & $\begin{array}{c}8.00 \\
(0.31)\end{array}$ & - & $\begin{array}{c}8.00 \\
(0.31)\end{array}$ \\
\hline (ii) & Transportation & $\begin{array}{l}20.00 \\
(0.78)\end{array}$ & $\begin{array}{l}20.00 \\
(0.77)\end{array}$ & - & $\begin{array}{l}20.00 \\
(0.78)\end{array}$ \\
\hline (iii) & Loading and unloading & $\begin{array}{l}20.00 \\
(0.78)\end{array}$ & $\begin{array}{l}20.00 \\
(0.77)\end{array}$ & - & $\begin{array}{l}20.00 \\
(0.78)\end{array}$ \\
\hline (iv) & Losses & $\begin{array}{l}14.00 \\
(0.54)\end{array}$ & $\begin{array}{l}20.00 \\
(0.77)\end{array}$ & - & $\begin{array}{l}17.00 \\
(0.66)\end{array}$ \\
\hline$(\mathrm{V})$ & Total cost incurred by producer & $\begin{array}{l}62.00 \\
(2.43)\end{array}$ & $\begin{array}{l}68.00 \\
(2.64)\end{array}$ & - & $\begin{array}{l}65.00 \\
(2.54)\end{array}$ \\
\hline & $\begin{array}{l}\text { Producer sale price consumer purchase } \\
\text { price }\end{array}$ & $\begin{array}{l}2547.00 \\
(100.00)\end{array}$ & $\begin{array}{l}2568.00 \\
(100.00)\end{array}$ & - & $\begin{array}{l}2557.50 \\
(100.00)\end{array}$ \\
\hline & Price spread & $\begin{array}{l}62.00 \\
(2.43)\end{array}$ & $\begin{array}{l}68.00 \\
(2.64)\end{array}$ & - & $\begin{array}{l}65.00 \\
(2.54)\end{array}$ \\
\hline
\end{tabular}


Note- Fingers in parenthesis show the percent to corresponding consumer's price

Channel-II (Producer-Retailer-Consumer):-

The price spread of ridged gourd was made through channel II ( producer-retailer-consumer). On an average, share in consumer's rupee was worked i.e. 65.66 per cent, which was comparatively lower than Channel-I because of one middleman i.e. retailer involved. Expenses incurred on marketing of ridged gourd and margins received by retailer came to 9.87 and 22.77 per cent, respectively. Per quintal price received by marginal, small and medium farmers were Rs. 2485, 2500 and 2600 respectively however producers share in consumer's rupee were 65.79, 65.82, and 65.40 per cent, respectively. On an average gross price spread was exhibited as 34.33 per cent (Table 5).

Table 5:-Price spread for Ridged gourd marketing in channel- II

\begin{tabular}{|c|c|c|c|c|c|}
\hline \multirow[t]{2}{*}{ S.No } & \multirow[t]{2}{*}{ Particulars } & \multicolumn{4}{|c|}{ Size of farms } \\
\hline & & Marginal & Small & Medium & Average \\
\hline 1. & $\begin{array}{l}\text { Net price received by the } \\
\text { producer }\end{array}$ & $\begin{array}{c}2485.00 \\
(65.79)\end{array}$ & $\begin{array}{l}2500.00 \\
(65.82)\end{array}$ & $\begin{array}{l}2600.00 \\
(65.40)\end{array}$ & $\begin{array}{l}2528.00 \\
(65.66)\end{array}$ \\
\hline 2. & \multicolumn{5}{|l|}{ Cost incurred by the producer } \\
\hline (i) & $\begin{array}{l}\text { Grading, filling, stretching, } \\
\text { packing etc }\end{array}$ & $\begin{array}{l}20.00 \\
(0.52)\end{array}$ & $\begin{array}{l}20.00 \\
(0.52)\end{array}$ & $\begin{array}{l}20.00 \\
(0.50)\end{array}$ & $\begin{array}{l}20.00 \\
(0.51)\end{array}$ \\
\hline (ii) & Transportation & $\begin{array}{l}20.00 \\
(0.52)\end{array}$ & $\begin{array}{l}20.00 \\
(0.52)\end{array}$ & $\begin{array}{l}20.00 \\
(0.50)\end{array}$ & $\begin{array}{l}20.00 \\
(0.51)\end{array}$ \\
\hline (iii) & Loading and unloading & $\begin{array}{l}20.00 \\
(0.52)\end{array}$ & $\begin{array}{l}20.00 \\
(0.52)\end{array}$ & $\begin{array}{l}20.00 \\
(0.50)\end{array}$ & $\begin{array}{l}20.00 \\
(0.51)\end{array}$ \\
\hline (iv) & Losses & $\begin{array}{l}15.00 \\
(0.39)\end{array}$ & $\begin{array}{l}15.00 \\
(0.39)\end{array}$ & $\begin{array}{l}15.00 \\
(0.37)\end{array}$ & $\begin{array}{l}15.00 \\
(0.38)\end{array}$ \\
\hline (v) & Total cost incurred by producer & $\begin{array}{l}65.00 \\
(1.72)\end{array}$ & $\begin{array}{l}65.00 \\
(1.71)\end{array}$ & $\begin{array}{l}65.00 \\
(1.63)\end{array}$ & $\begin{array}{l}65.00 \\
(1.68)\end{array}$ \\
\hline (vi) & $\begin{array}{l}\text { Producer sale price retailer } \\
\text { purchase price }\end{array}$ & $\begin{array}{c}2550.00 \\
(66.51) \\
\end{array}$ & $\begin{array}{l}2565.00 \\
(67.53) \\
\end{array}$ & $\begin{array}{c}2665.00 \\
(67.04) \\
\end{array}$ & $\begin{array}{l}2593.00 \\
(67.35) \\
\end{array}$ \\
\hline (3) & \multicolumn{5}{|l|}{ Cost insured by retailer } \\
\hline (i) & Market fee & $\begin{array}{l}50.00 \\
(1.32)\end{array}$ & $\begin{array}{l}50.00 \\
(1.31)\end{array}$ & $\begin{array}{l}52.00 \\
(1.30)\end{array}$ & $\begin{array}{l}51.00 \\
(1.32)\end{array}$ \\
\hline (ii) & Commission & $\begin{array}{l}150.00 \\
(3.97)\end{array}$ & $\begin{array}{l}150.00 \\
(3.94)\end{array}$ & $\begin{array}{l}156.00 \\
(3.92)\end{array}$ & $\begin{array}{l}152.00 \\
(3.94)\end{array}$ \\
\hline (iii) & Transportation & $\begin{array}{l}20.00 \\
(0.52)\end{array}$ & $\begin{array}{l}20.00 \\
(0.52)\end{array}$ & $\begin{array}{l}20.00 \\
(0.50)\end{array}$ & $\begin{array}{l}20.00 \\
(0.51)\end{array}$ \\
\hline (iv) & Rent of shop/rehire & $\begin{array}{l}50.00 \\
(1.32)\end{array}$ & $\begin{array}{l}50.00 \\
(1.31)\end{array}$ & $\begin{array}{l}50.00 \\
(1.25)\end{array}$ & $\begin{array}{l}50.00 \\
(1.29)\end{array}$ \\
\hline (v) & Loss wastage and spoilage & $\begin{array}{l}102.00 \\
(2.70)\end{array}$ & $\begin{array}{l}108.00 \\
(2.84)\end{array}$ & $\begin{array}{l}112.00 \\
(2.81)\end{array}$ & $\begin{array}{l}107.00 \\
(2.77)\end{array}$ \\
\hline (vi) & Total cost incurred by retailer & $\begin{array}{l}372.00 \\
(9.84)\end{array}$ & $\begin{array}{l}378.00 \\
(9.95)\end{array}$ & $\begin{array}{l}390.00 \\
(9.81)\end{array}$ & $\begin{array}{c}380.00 \\
(9.87)\end{array}$ \\
\hline (4) & Retailer margin & $\begin{array}{l}855.00 \\
(22.63)\end{array}$ & $\begin{array}{l}855.00 \\
(22.51)\end{array}$ & $\begin{array}{l}920.00 \\
(23.14)\end{array}$ & $\begin{array}{l}877.00 \\
(22.77)\end{array}$ \\
\hline (5) & $\begin{array}{l}\text { Retailer sale price Consumer } \\
\text { purchase price }\end{array}$ & $\begin{array}{l}3777.00 \\
(100.00)\end{array}$ & $\begin{array}{l}3798.00 \\
(100.00)\end{array}$ & $\begin{array}{l}3975.00 \\
(100.00)\end{array}$ & $\begin{array}{l}3850.00 \\
(100.00)\end{array}$ \\
\hline & Price spread & $\begin{array}{l}1292.00 \\
(34.20)\end{array}$ & $\begin{array}{l}1298.00 \\
(34.17)\end{array}$ & $\begin{array}{c}1375.00 \\
(34.59)\end{array}$ & $\begin{array}{l}1322.00 \\
(34.33)\end{array}$ \\
\hline
\end{tabular}

Note- Fingers in parenthesis show the percent to corresponding consumer's price

\section{Channel-III (Producer- Wholesaler-Retailer-Consumer):-}

On an average, share in consumer's rupee was worked i.e. 63.72 per cent, which was comparatively lower than Channel-II because of two middlemen's i.e. wholesaler and retailer were involved. Expenses incurred on marketing costs and margins received by wholesaler were 5.09 per cent and 2.44 per cent, respectively, whereas expenses incurred on marketing costs and margins received by retailer were 4.58 and 21.42 per cent, respectively. Per quintal price received by marginal, small and medium farmers were Rs.2485, 2500 and 2600, however; producer's share in 
consumer's rupee came to $64.83,64.20$ and 63.78 per cent, respectively. On an overall average gross price spread was exhibited 35.44 per cent (Table 6).

Table6:- Price spread for Ridged gourd marketing in channel-III (Rs./qt.)

\begin{tabular}{|c|c|c|c|c|c|}
\hline \multirow{2}{*}{$\begin{array}{l}\text { S. } \\
\text { No. }\end{array}$} & \multirow[t]{2}{*}{ Particulars } & \multicolumn{4}{|c|}{ Size of farms } \\
\hline & & Marginal & Small & Medium & Average \\
\hline 1 & Net price received by the producer & $\begin{array}{c}2485.00 \\
(64.83) \\
\end{array}$ & $\begin{array}{r}2500.00 \\
(64.20) \\
\end{array}$ & $\begin{array}{c}2600.00 \\
(63.78) \\
\end{array}$ & $\begin{array}{r}2528.00 \\
(63.72) \\
\end{array}$ \\
\hline 2 & \multicolumn{5}{|l|}{ Costs incurred by the producer } \\
\hline (i) & Grading, filling, stretching, packing etc. & $\begin{array}{l}20.00 \\
(0.52)\end{array}$ & $\begin{array}{l}20.00 \\
(0.51)\end{array}$ & $\begin{array}{l}20.00 \\
(0.49)\end{array}$ & $\begin{array}{l}20.00 \\
(0.50)\end{array}$ \\
\hline (ii) & Transportation. & $\begin{array}{l}20.00 \\
(0.52)\end{array}$ & $\begin{array}{l}20.00 \\
(0.51)\end{array}$ & $\begin{array}{l}20.00 \\
(0.49)\end{array}$ & $\begin{array}{l}20.00 \\
(0.50)\end{array}$ \\
\hline (iii) & Loading unloading. & $\begin{array}{l}20.00 \\
(0.52)\end{array}$ & $\begin{array}{l}20.00 \\
(0.51)\end{array}$ & $\begin{array}{l}20.00 \\
(0.49)\end{array}$ & $\begin{array}{l}20.00 \\
(0.50)\end{array}$ \\
\hline (iv) & Losses and wastage. & $\begin{array}{l}15.00 \\
(0.39)\end{array}$ & $\begin{array}{l}15.00 \\
(0.38)\end{array}$ & $\begin{array}{l}15.00 \\
(0.36)\end{array}$ & $\begin{array}{l}15.00 \\
(0.37)\end{array}$ \\
\hline (v) & Total cost incurred by producer & $\begin{array}{l}65.00 \\
(1.69) \\
\end{array}$ & $\begin{array}{l}65.00 \\
(1.66)\end{array}$ & $\begin{array}{l}65.00 \\
(1.59) \\
\end{array}$ & $\begin{array}{l}65.00 \\
(1.63)\end{array}$ \\
\hline (vi) & $\begin{array}{l}\text { Purchase sale price Commission agent / whole } \\
\text { seller's purchase price }\end{array}$ & $\begin{array}{c}2550.00 \\
(66.52)\end{array}$ & $\begin{array}{r}2565.00 \\
(65.87)\end{array}$ & $\begin{array}{c}2665.00 \\
(65.38)\end{array}$ & $\begin{array}{c}2593.00 \\
(65.36)\end{array}$ \\
\hline 3 & \multicolumn{5}{|c|}{ Cost incurred by the whole seller/Commission agent } \\
\hline (i) & Market fee & $\begin{array}{l}50.00 \\
(1.30)\end{array}$ & $\begin{array}{l}50.00 \\
(1.28)\end{array}$ & $\begin{array}{l}52.00 \\
(1.27)\end{array}$ & $\begin{array}{l}51.00 \\
(1.28)\end{array}$ \\
\hline (ii) & Commission & $\begin{array}{l}150.00 \\
(3.91)\end{array}$ & $\begin{array}{c}150.00 \\
(3.85)\end{array}$ & $\begin{array}{l}156.00 \\
(3.82)\end{array}$ & $\begin{array}{l}152.00 \\
(3.83)\end{array}$ \\
\hline (iii) & $\begin{array}{l}\text { Total cost incurred by Commission agent / } \\
\text { whole seller's }\end{array}$ & $\begin{array}{c}200.00 \\
(5.21)\end{array}$ & $\begin{array}{c}200.00 \\
(5.13)\end{array}$ & $\begin{array}{r}208.00 \\
(5.10)\end{array}$ & $\begin{array}{c}202.00 \\
(5.09)\end{array}$ \\
\hline (iv) & Commission agent / whole seller's margin. & $\begin{array}{l}97.00 \\
(2.53)\end{array}$ & $\begin{array}{l}97.00 \\
(2.49)\end{array}$ & $\begin{array}{l}97.00 \\
(2.37)\end{array}$ & $\begin{array}{l}97.00 \\
(2.44)\end{array}$ \\
\hline (v) & $\begin{array}{l}\text { Commission agent / whole seller's sale price } \\
\text { retailer purchase price. }\end{array}$ & $\begin{array}{c}2847.00 \\
(74.27)\end{array}$ & $\begin{array}{l}2862.00 \\
(73.49)\end{array}$ & $\begin{array}{c}2970.00 \\
(72.86)\end{array}$ & $\begin{array}{r}2893.00 \\
(72.92) \\
\end{array}$ \\
\hline 4 & \multicolumn{5}{|l|}{ Cost incurred by retailer } \\
\hline (i) & Transportation & $\begin{array}{l}20.00 \\
(0.52)\end{array}$ & $\begin{array}{l}20.00 \\
(0.51)\end{array}$ & $\begin{array}{l}20.00 \\
(0.49)\end{array}$ & $\begin{array}{l}20.00 \\
(0.50)\end{array}$ \\
\hline (ii) & Labour charge & $\begin{array}{c}8.00 \\
(0.20)\end{array}$ & $\begin{array}{c}8.00 \\
(0.20)\end{array}$ & $\begin{array}{c}8.00 \\
(0.19)\end{array}$ & $\begin{array}{c}8.00 \\
(0.20)\end{array}$ \\
\hline (iii) & Rent of shop/rehire & $\begin{array}{l}50.00 \\
(1.30)\end{array}$ & $\begin{array}{l}50.00 \\
(1.28)\end{array}$ & $\begin{array}{l}50.00 \\
(1.22)\end{array}$ & $\begin{array}{l}50.00 \\
(1.26)\end{array}$ \\
\hline (iv) & Loss wastage and spoilage & $\begin{array}{l}108.00 \\
(2.81)\end{array}$ & $\begin{array}{l}114.00 \\
(2.92)\end{array}$ & $\begin{array}{l}118.00 \\
(2.89)\end{array}$ & $\begin{array}{l}104.00 \\
(2.62)\end{array}$ \\
\hline (v) & Total cost incurred by retailer & $\begin{array}{l}186.00 \\
(4.85) \\
\end{array}$ & $\begin{array}{l}192.00 \\
(4.93)\end{array}$ & $\begin{array}{l}196.00 \\
(4.80)\end{array}$ & $\begin{array}{l}182.00 \\
(4.58) \\
\end{array}$ \\
\hline (vi) & Retailer margin & $\begin{array}{l}800.00 \\
(20.87)\end{array}$ & $\begin{array}{l}840.00 \\
(21.57)\end{array}$ & $\begin{array}{l}910.00 \\
(22.32)\end{array}$ & $\begin{array}{l}850.00 \\
(21.42)\end{array}$ \\
\hline (vii) & Retailer sale price Consumer purchase price & $\begin{array}{l}3833.00 \\
(100.00)\end{array}$ & $\begin{array}{l}3894.00 \\
(100.00)\end{array}$ & $\begin{array}{l}4076.00 \\
(100.00)\end{array}$ & $\begin{array}{c}3967.00 \\
(100.00)\end{array}$ \\
\hline & Price spread & $\begin{array}{l}1348.00 \\
(35.16)\end{array}$ & $\begin{array}{l}1394.00 \\
(35.79)\end{array}$ & $\begin{array}{c}1476.00 \\
(36.21)\end{array}$ & $\begin{array}{c}1406.00 \\
(35.44)\end{array}$ \\
\hline
\end{tabular}

Note: Figures in parenthesis show per cent to corresponding consumer's price.

\section{Inter-channel comparison as a whole for Ridged gourd:-}

Table 7 highlights summary of inter-channel comparison in respect of average marketing costs, margins and price spread of ridged gourd. It is interesting to mention that marketing costs increased as increase in number of intermediaries under Channel-II and Channel-III. On comparing, gross marketing margins was found maximum 
having 35.44 per cent in Channel-III followed by 34.33 per cent and 2.54 per cent in Channel-II and Channel-I, respectively.

Table7:- Inter-channel comparison as a whole for Ridged gourd (Rs./qt.)

\begin{tabular}{|c|c|c|c|c|}
\hline S. No. & Particulars & $\begin{array}{c}\text { Channel- } \\
\text { I }\end{array}$ & Channel-II & Channel-III \\
\hline 1. & Net price received by the producer & $\begin{array}{c}2492.50 \\
(97.45)\end{array}$ & $\begin{array}{l}2528.00 \\
(65.66)\end{array}$ & $\begin{array}{c}2528.00 \\
(63.72)\end{array}$ \\
\hline 2. & \multicolumn{4}{|l|}{ Cost incurred by the producer } \\
\hline (i) & Grading, filling weighing, stretching etc & $\begin{array}{c}8.00 \\
(0.31)\end{array}$ & $\begin{array}{l}20.00 \\
(0.51)\end{array}$ & $\begin{array}{l}20.00 \\
(0.50)\end{array}$ \\
\hline (ii) & Transportation & $\begin{array}{l}20.00 \\
(0.78)\end{array}$ & $\begin{array}{l}20.00 \\
(0.51)\end{array}$ & $\begin{array}{l}20.00 \\
(0.50)\end{array}$ \\
\hline (iii) & Loading and unloading & $\begin{array}{l}20.00 \\
(0.78)\end{array}$ & $\begin{array}{l}20.00 \\
(0.51)\end{array}$ & $\begin{array}{l}20.00 \\
(0.50)\end{array}$ \\
\hline (iv) & Losses & $\begin{array}{l}17.00 \\
(0.66)\end{array}$ & $\begin{array}{l}15.00 \\
(0.38)\end{array}$ & $\begin{array}{l}15.00 \\
(0.37)\end{array}$ \\
\hline (v) & Total cost incurred by producer & $\begin{array}{l}65.00 \\
(2.54)\end{array}$ & $\begin{array}{c}65.00 \\
(1.68)\end{array}$ & $\begin{array}{l}65.00 \\
(1.63)\end{array}$ \\
\hline \multirow[t]{2}{*}{ (vi) } & Producer sale price consumer purchase price & $\begin{array}{c}2561 \\
(100.00)\end{array}$ & $\begin{array}{c}2593.00 \\
(67.35)\end{array}$ & $\begin{array}{c}2593.00 \\
(65.36)\end{array}$ \\
\hline & \multicolumn{4}{|l|}{ Cost insured by retailer } \\
\hline (i) & Market fee & - & $\begin{array}{l}51.00 \\
(1.32)\end{array}$ & - \\
\hline (ii) & Commission & - & $\begin{array}{l}152.00 \\
(3.94)\end{array}$ & - \\
\hline (iii) & Transportation & - & $\begin{array}{l}20.00 \\
(0.51)\end{array}$ & - \\
\hline (iv) & Rent of shop/rehire & - & $\begin{array}{l}50.00 \\
(1.29)\end{array}$ & - \\
\hline (v) & Loss wastage and spoilage & - & $\begin{array}{l}107.00 \\
(2.77)\end{array}$ & - \\
\hline (vi) & Total cost incurred by retailer & - & $\begin{array}{c}380.00 \\
(9.87)\end{array}$ & - \\
\hline (vii) & Retailer margin & - & $\begin{array}{l}877.00 \\
(22.77) \\
\end{array}$ & - \\
\hline (Viii) & Retailer sale price Consumer purchase price & - & $\begin{array}{l}3850.00 \\
(100.00)\end{array}$ & - \\
\hline \multicolumn{5}{|c|}{ Total cot incurred by whole seller/commission agent } \\
\hline (i) & Market fee & - & - & $\begin{array}{l}51.00 \\
(1.28)\end{array}$ \\
\hline (ii) & Commission & - & - & $\begin{array}{l}152.00 \\
(3.83)\end{array}$ \\
\hline (iii) & Total cost incurred by Commission agent / whole seller's & - & - & $\begin{array}{c}202.00 \\
(5.09)\end{array}$ \\
\hline (iv) & Commission agent / whole seller's margin. & - & - & $\begin{array}{l}97.00 \\
(2.44)\end{array}$ \\
\hline \multirow[t]{2}{*}{ (v) } & $\begin{array}{l}\text { Commission agent / whole seller's sale price retailer } \\
\text { purchase price. }\end{array}$ & - & - & $\begin{array}{c}2893.00 \\
(72.92)\end{array}$ \\
\hline & \multicolumn{4}{|l|}{ Cost incurred by retailer } \\
\hline (i) & Transportation & - & - & $\begin{array}{l}20.00 \\
(0.50)\end{array}$ \\
\hline (ii) & Labour charge & - & - & $\begin{array}{c}8.00 \\
(0.20)\end{array}$ \\
\hline (iii) & Rent of shop/rehire & - & - & 50.00 \\
\hline
\end{tabular}




\begin{tabular}{|c|l|c|c|c|}
\hline & & & & $(1.26)$ \\
\hline (iv) & Loss wastage and spoilage & - & - & $\begin{array}{c}104.00 \\
(2.62)\end{array}$ \\
\hline (v) & Total cost incurred by retailer & - & - & $\begin{array}{c}182.00 \\
(4.58)\end{array}$ \\
\hline (vi) & Retailer margin & - & - & $\begin{array}{c}850.00 \\
(21.42)\end{array}$ \\
\hline (vii) & Retailer sale price Consumer purchase price & & & 3967.00 \\
& & - & - & $(100.00)$ \\
\hline & Price spread & 65.00 & 1322.00 & 1406.00 \\
& & $(2.54)$ & $(34.33)$ & $(35.44)$ \\
\hline
\end{tabular}

Note: Figures in parenthesis show per cent to corresponding consumer's price.

\section{Marketing efficiency of Ridged gourd:-}

Marketing efficiency moves around the fact that what extent of market agencies has been able to move the goods at minimum cost extending the maximum services from the producer to final consumer. Table 8 revealed that, Channel-I was found more efficient as compared to Channel- II, and Channel-III because there was no involvement of any middleman and produce was sold directly to the consumers which resulted less marketing cost in Channel-I compared to other channels. It could be observed that marketing efficiency has inverse relationship with the total cost and margins. As number of intermediaries increased the cost and margins increased, the cost and margin increased and the inverse has been the marketing efficiency (Balappa and Hugas, 2003 and Sekhon et. al. 2015)

Table8:-Marketing efficiency of Ridged gourd in different channel.

\begin{tabular}{|c|c|c|c|}
\hline Channel & $\begin{array}{c}\text { Value of ridged gourd sold (Rs. /q.) } \\
\text { (consumer's price) }\end{array}$ & $\begin{array}{c}\text { Gross marketing } \\
\text { margin (Rs./q.) }\end{array}$ & Marketing efficiency \\
\hline I & 2557.00 & 65.00 & 39.33 \\
\hline II & 3901.00 & 1322.00 & 2.95 \\
\hline III & 3976.00 & 1406.00 & 2.82 \\
\hline
\end{tabular}

\section{Constraints in production and marketing:-}

The major problems faced by vegetables growers in study area were analyzed, and presented in Table 9. It was observed during investigation that technical problems were most serious in production of vegetables. Technical problems were highest on medium farms ( 72.89 percent) followed by small farms and marginal farms. Financial problems were second rank and it was highest on small farms (69.30 per cent) followed by marginal farms and medium farms. Miscellaneous problems (losses and ricks) rank third for vegetables. In considering size group of farms, marginal farms constituted maximum (64.42 per cent) followed by small farms and medium farms, respectively. Management problems were found as the rank fifth and found highest on medium farms (64.67 per cent) followed by small farms and marginal farms whereas, for vegetables, marketing problems it was highest on marginal farms (63.08 per cent) followed by medium and small farms, respectively. On an average, for vegetables technical problems, financial problems, miscellaneous problems, marketing problems and management problems were $70.58,67.42,63.95,61.04$, and 43.85 per cent, respectively.

Table 9:-Constraints in production and marketing on different size of farms.

(Percentage)

\begin{tabular}{|c|l|c|c|c|c|c|}
\hline \multirow{2}{*}{ S. No } & \multicolumn{1}{|c|}{ Particulars } & \multicolumn{3}{|c|}{ Size of Farms } & Total & \multirow{2}{*}{ Rank } \\
\cline { 2 - 5 } & & Marginal & Small & Medium & & \\
\hline 1 & Technical problems & 69.29 & 72.50 & 72.89 & 70.58 & \multirow{2}{*}{ I } \\
\hline (i) & Related highly yield variety of seed & 77.46 & 75.33 & 75.00 & 76.60 \\
\hline (ii) & High incidence of disease/insects in HYVs & 76.81 & 81 & 60.00 & 76.56 \\
\hline (iii) & Low availability of power & 95.40 & 90.33 & 91.12 & 93.50 \\
\hline (iv) & $\begin{array}{l}\text { Lack of knowledge about recommended } \\
\text { package of practises }\end{array}$ & 27.50 & 43.4 & 65.56 & 35.70 & \\
\hline (2) & Management problems & 38.21 & 49.06 & 64.67 & 43.85 & \multirow{2}{*}{ V } \\
\hline (i) & Skilled person & 36.80 & 52.17 & 68.89 & 44.30 & \\
\hline (ii) & Trained person & 42.27 & 48.67 & 65.12 & 46.24 & \\
\hline (iii) & Quick decision person & 35.60 & 46.4 & 60.12 & 41.05 & \\
\hline
\end{tabular}




\begin{tabular}{|c|c|c|c|c|c|c|}
\hline 3 & Financial problems & 66.96 & 69.30 & 64.22 & 67.42 & \multirow[t]{6}{*}{ II } \\
\hline (i) & Adequacy problems & 81.03 & 77.27 & 49.78 & 77.09 & \\
\hline (ii) & Time lines & 57.34 & 61.73 & 63.89 & 59.25 & \\
\hline (iii) & Documents & 51.22 & 48.3 & 76.78 & 52.65 & \\
\hline (iv) & Bribes & 65.90 & 79.57 & 50.89 & 68.65 & \\
\hline (v) & Subsidy & 80.01 & 79.67 & 80.22 & 79.93 & \\
\hline 4 & Marketing problems & 63.08 & 57.5 & 59.00 & 61.04 & \multirow[t]{6}{*}{ IV } \\
\hline (i) & Processing & 78.80 & 63.63 & 51.89 & 71.83 & \\
\hline (ii) & Grading & 76.30 & 67.07 & 65.78 & 72.58 & \\
\hline (iii) & Transportation & 62.70 & 54.23 & 58.00 & 59.74 & \\
\hline (iv) & Storage & 77.42 & 74.53 & 67.78 & 75.69 & \\
\hline (v) & Unavailability of chemicals & 20.20 & 28.03 & 51.88 & 25.40 & \\
\hline 5 & Miscellaneous problems & 64.42 & 63.30 & 62.89 & 63.95 & \multirow[t]{4}{*}{ III } \\
\hline (i) & Losses & 65.70 & 50.93 & 60.22 & 60.78 & \\
\hline \multirow[t]{2}{*}{ (i) } & Risk & 63.14 & 75.7 & 65.66 & 67.14 & \\
\hline & Total farms & $\begin{array}{c}61 \\
(100.00)\end{array}$ & $\begin{array}{c}30 \\
(100.00)\end{array}$ & $\begin{array}{c}09 \\
(100.00)\end{array}$ & $\begin{array}{c}100.00 \\
(100.00)\end{array}$ & \\
\hline
\end{tabular}

Note- Figure in parentheses shows the percent to corresponding total score.

\section{Summary and Conclusions:-}

The study concluded that ridge gourd cultivation was a remunerative enterprise in study area and give positive returns to farmers. Overall average cost of cultivation was worked out to be Rs. 117872. The cost of cultivation was maximum on medium farms fallowed by marginal and small farms. On an average the study further reveals that major components on which maximum cost was incurred on human labour followed by plant protection, seed, manures and fertilizer, irrigation, machinery charges and bullock labour, respectively. Input-output ratio related to $\operatorname{cost} \mathrm{C}_{3}$ was highest on medium farms (1.85) followed by small farms and marginal farms. The elasticity of production under marginal, small and medium farms with respect to human labour and seeds were statistically significant whereas, under marginal farms, manure \& fertilizers were found statistically significance respectively. Returns to scale indicates decreasing return to scale. The marketing efficiency under that channel-I was found to be highest as compared to channel-II and channel-III, because no middleman was found in channel-I. The producer's share in consumer's rupee was found maximum in channel-I followed by channel-II and channel-III, respectively.

Suggestive policy implications to overcome the production and marketing constraints of vegetables are that farmers be trained through designated extension agency/services by participation directly and updated about the latest farming technology and management of vegetables crop. Input supply should be linked with co-operative marketing system to help the producers \& traders. Farm advisory services should be available at Block head quarter to improve the close contact between farmers and extension workers. Farm advisory services should be available at Block head quarter to improve the close contact within farmers and extension workers. Besides these measures improvement in market infrasture such as storage facilities, cold storage, refrigerated van and better road links etc.; improve marketing efficiency (Gandhi et. al). Need to strengthen the market information system through the internet facilities and other means of communications.

\section{Bibliography:-}

1. Ali, J. and Kapoor, S.(2008): Farmers' perception on risks in fruits and vegetables production: An empirical study of Uttar Pradesh. Agricultural Economics Research Review., 21:317-326

2. Baba, S.H., and Mann, A.S.(2005) :Resource use efficiency of main and off-season vegetables under irrigated condition of Himachal Pradesh. Indian Journal of Agricultural Economics., 60(3): 533-534.

3. Balappa, S.R., and Hugus, L.B. (2003) An economic evaluation of onion production and its marketing system in Karnataka.Indian Journal of Agricultural Marketing.46 (2):22-24

4. Gandhi, V.P. and Nambodiri, N.V. (2008): Fruits and vegetable marketing in India. Glimpses of Indian Agriculture Macro and Micro Aspects Volume., 1:403-406.

5. Joshi, P.K.,Joshi, L. and Birthal,P.S.(2006): Diversification and its impact on smallholders: evidence from a study on vegetable production. Agricultural Economics Research Review., 19: 219-236.

6. Sekhon, M.K., Sindhu, M.S., Kaur, M. and Kaur, P.(2015): Cost-returns analysis and marketing pattern of summer maize in Punjab. Indian Journal of Agricultural Marketing., 29(1)31-42. 
7. Shrestha, R.B., Huang,W.C., and Pradhan., U. (2015): Resource use efficiency in vegetable production in the high hills of Eastern Nepal, Nepalese Journal of Agricultural Economics., 2-3:79-87

8. Singh, G., Sharma, V. K. and Singh, S. (2015). Production and marketing of green peas in Punjab. Indian Journal of Agricultural Marketing., 29(1): 71-80

9. Singh, M.K.(1997): Economics of production and marketing of vegetables: a case study Jabalpur district. Agricultural Marketing., 40(2):18-20.

in Patan block of 